\title{
Mapping the nomenclature, methodology, and reporting of studies that review methods: a pilot methodological review
}

\author{
Daeria O. Lawson ${ }^{1 *}$, Alvin Leenus ${ }^{2}$ and Lawrence Mbuagbaw ${ }^{1,3}$
}

\begin{abstract}
Background: A relatively novel method of appraisal, methodological reviews (MRs) are used to synthesize information on the methods used in health research. There are currently no guidelines available to inform the reporting of MRs.
\end{abstract}

Objectives: This pilot review aimed to determine the feasibility of a full review and the need for reporting guidance for methodological reviews.

Methods: Search strategy: We conducted a search of PubMed, restricted to 2017 to include the most recently published studies, using different search terms often used to describe methodological reviews: "literature survey" OR "meta-epidemiologic* review" OR "meta-epidemiologic* survey" OR "methodologic* review" OR "methodologic* survey" OR "systematic survey."

Data extraction: Study characteristics including country, nomenclature, number of included studies, search strategy, a priori protocol use, and sampling methods were extracted in duplicate and summarized.

Outcomes: Primary feasibility outcomes were the sensitivity and specificity of the search terms (criteria for success of feasibility set at sensitivity and specificity of $\geq 70 \%$ ).

Analysis: The estimates are reported as a point estimate ( $95 \%$ confidence interval).

Results: Two hundred thirty-six articles were retrieved and 31 were included in the final analysis. The most accurate search term was "meta-epidemiological" (sensitivity [Sn] 48.39; 95\% Cl 31.97-65.16; specificity [Sp] 97.56; 94.4298.95). The majority of studies were published by authors from Canada $(n=12,38.7 \%)$, and Japan and USA ( $n=4$, 12.9\% each). The median (interquartile range [IQR]) number of included studies in the MRs was 77 (13-1127). Reporting of a search strategy was done in most studies $(n=23,74.2 \%)$. The use of a pre-published protocol ( $n=7,22.6 \%)$ or a justifiable sampling method $(n=5,16.1 \%)$ occurred rarely.

Conclusions: Using the MR nomenclature identified, it is feasible to build a comprehensive search strategy and conduct a full review. Given the variation in reporting practices and nomenclature attributed to MRs, there is a need for guidance on standardized and transparent reporting of MRs. Future guideline development would likely include stakeholders from Canada, USA, and Japan.

Keywords: Feasibility, Guidelines, Methodological review, Nomenclature, Pilot, Reporting

\footnotetext{
* Correspondence: lawsod3@mcmaster.ca

${ }^{1}$ Department of Health Research Methods, Evidence, and Impact, McMaster

University, 1280 Main Street West, Hamilton, ON L8S 4K1, Canada

Full list of author information is available at the end of the article
}

(c) The Author(s). 2020 Open Access This article is distributed under the terms of the Creative Commons Attribution 4.0 International License (http://creativecommons.org/licenses/by/4.0/), which permits unrestricted use, distribution, and reproduction in any medium, provided you give appropriate credit to the original author(s) and the source, provide a link to the Creative Commons license, and indicate if changes were made. The Creative Commons Public Domain Dedication waiver (http://creativecommons.org/publicdomain/zero/1.0/) applies to the data made available in this article, unless otherwise stated. 


\section{Background}

Health researchers, methodologists, and policymakers rely on primary studies or evidence syntheses (e.g., systematic reviews) to provide summaries of evidence for decisionmaking $[1,2]$. However, the credibility of this evidence depends on how the studies were conducted and reported. Therefore, critical appraisal of health research methodology is an important tool for researchers and end-users of evidence. As such, certain studies exist solely to help synthesize methodological data about the design, analysis, and reporting of primary and secondary research. These studies can be referred to, for the purposes of this paper, as methodological reviews (MRs) and represent an efficient way of assessing research methods and summarizing methodological issues in the conduct, analysis, and reporting of health research. Collating primary and secondary research in this way can help to identify reporting and methodological gaps, generate empirical evidence on the state of or quality of conduct and reporting, and inform the development of reporting and methodological standards. MRs are highly informative because they allow researchers to evaluate study methods; assess adherence, quality, and completeness of reporting (e.g., reporting adherence to Consolidated Standards of Reporting Trials, CONSORT); document and assess the variety of methods used or approaches to analyses (e.g., statistical approaches for handling missing data in cluster randomized trials); demonstrate changes in reporting over time (e.g., since the introduction of a specific guideline); demonstrate consistency between study abstracts/trial registries and their full texts; and many other issues [3-8]. In this way, MRs are indispensable to high-quality health research by allowing researchers to identify inappropriate research method practices and propose solutions.

Reporting guidelines are important tools in improving the reporting and conduct of health research, and many exist for various study designs. Currently, the Enhancing the QUAlity and Transparency Of health Research (EQUATOR) network is the leading authority in reporting guidelines for health research [9]. As of September 2019, this website lists 418 guidelines, with another 74 currently under development. There is empirical evidence that publication of reporting guidelines improves reporting, but this is often contingent on journal endorsement as well as the period of time since publication [10-12]. However, there is no published guidance for reporting methodological reviews despite an increase in their development and usage [13].

Murad and Wang have proposed a checklist which is an adaptation of the Preferred Reporting Items for Systematic Reviews and Meta-Analyses (PRISMA), a widely endorsed guideline developed to help report systematic reviews [14, 15]. While its use would ensure that a standardized and transparent approach in reporting is followed, this does not address the various typologies of MRs including the variety of approaches used to conduct these studies. For example,
MRs that use a before-after design, interrupted time series, or random sampling approaches would be a poor fit for this tool $[7,16-18]$. Likewise, studies in which the unit of analysis is not the "study" would require more specific guidance (e.g., some MRs investigate multiple subgroup analyses within the same study) $[18,19]$. Further, some MRs report formal sample size estimations, and it is unclear whether this should be recommended for all MRs. In line with this thinking, recent correspondences with the Journal of Clinical Epidemiology highlight that methodological studies cannot always be classified as systematic reviews, but instead represent their own branch of evidence synthesis methodology requiring specially tailored reporting guidance [20].

To facilitate the development of reporting guidelines for health research, Moher et al. propose a five-phase, 18-step strategy [10]. One important step in this process is a review of the literature, including seeking evidence on the quality of published research articles and identifying information related to sources of bias in reporting. However, some concerns exist with reviewing the literature. First, the literature on methodological reviews is elusive and can be found in any journal or database. Second, given the relative novelty and rapid development of the field, there is no formally accepted nomenclature to guide a literature search. We therefore deemed it necessary to conduct a pilot methodological review of methodological reviews to inform the feasibility of a broader, full methodological review based on our ability to

1. Determine the appropriate nomenclature for identification of methodological reviews

2. Determine a preliminary need for guidance, based on inconsistencies in reporting

Pilot studies of research syntheses, contrary to pilot studies of classical "primary" research, aim to: establish the need for a full review, establish the value of the methods used, and to identify, clarify, and review any problems with the processes and instruments. They can also be used to identify conceptual, methodological, and practical problems that need to be addressed in a full review. In this way, piloting research syntheses maximizes validity and efficiency [21, 22]. The research questions we sought to answer in this pilot review, where they fit in the larger scheme of the project objectives, and their implications for a full review (i.e., larger study to further explore the observed methodological variations in a broader sample of MRs) are outlined in Table 1. The listed research questions were evaluated in and applied to the MRs included in this pilot.

\section{Methods}

Study design

The methods reported are in line with current guidance on piloting evidence syntheses, by way of a "mini-review" all the way through [21]. We conducted a pilot 
Table 1 Pilot research questions and implications for a full review

\begin{tabular}{|c|c|c|c|}
\hline Pilot review objectives & Research questions & Implications for feasibility of full review & Metrics/threshold \\
\hline $\begin{array}{l}\text { Determine the appropriate } \\
\text { nomenclature for accurate } \\
\text { identification of methodological } \\
\text { reviews }\end{array}$ & $\begin{array}{l}\text { Which search terms } \\
\text { yield methodological } \\
\text { reviews? }\end{array}$ & $\begin{array}{l}\text { Identifying a list of terms that yields } \\
\text { methodological reviews will inform the } \\
\text { search strategy in the full review }\end{array}$ & Sensitivity/specificity $\geq 70 \%$ \\
\hline \multirow[t]{7}{*}{$\begin{array}{l}\text { Determine the need for } \\
\text { methodological review reporting } \\
\text { guidelines }\end{array}$} & $\begin{array}{l}\text { Are research } \\
\text { methods specified a } \\
\text { priori? }\end{array}$ & $\begin{array}{l}\text { Inconsistent pre-specification of methods } \\
\text { would indicate the need for a full review }\end{array}$ & $\leq 70 \%$ with published protocols \\
\hline & $\begin{array}{l}\text { How many databases } \\
\text { are searched? }\end{array}$ & $\begin{array}{l}\text { Wide variation in the numbers of databases } \\
\text { searched would indicate the need for a full } \\
\text { review }\end{array}$ & $\begin{array}{l}\text { Coefficient of variation } \sim 1 \text { (i.e., } \\
\text { spread in results relative to the mean) }\end{array}$ \\
\hline & $\begin{array}{l}\text { Are search time limits } \\
\text { justified? }\end{array}$ & $\begin{array}{l}\text { Inappropriate justification of search time } \\
\text { limits would imply the need for a full review }\end{array}$ & $\leq 70 \%$ justify search limits \\
\hline & $\begin{array}{l}\text { Is the sample size } \\
\text { justified? }\end{array}$ & $\begin{array}{l}\text { Inappropriate justification of sample size for } \\
\text { MRs designed as analytical studies (e.g., } \\
\text { before-after comparisons, regression-based } \\
\text { analyses) would imply the need for a full } \\
\text { review }\end{array}$ & $\begin{array}{l}\leq 70 \% \text { justify sample size or perform } \\
\text { sample size calculation }\end{array}$ \\
\hline & $\begin{array}{l}\text { Is a formal sample size } \\
\text { calculation performed? }\end{array}$ & $\begin{array}{l}\text { Inappropriate justification of sample size for } \\
\text { MRs designed as analytical studies (e.g., before- } \\
\text { after comparisons, regression-based analyses) } \\
\text { would imply the need for a full review }\end{array}$ & \\
\hline & $\begin{array}{l}\text { Is a random sample of } \\
\text { studies used? }\end{array}$ & $\begin{array}{l}\text { Use of different sampling approaches to select } \\
\text { a subset of studies from a larger group would } \\
\text { indicate the need for a full review }\end{array}$ & $\begin{array}{l}\text { Among studies where the goal was } \\
\text { not to capture all available studies, } \\
\leq 70 \% \text { use a random sampling approach }\end{array}$ \\
\hline & $\begin{array}{l}\text { Do research methods } \\
\text { or authors suggest } \\
\text { generalizable findings? }\end{array}$ & $\begin{array}{l}\text { Lack of clear approaches to reporting } \\
\text { generalizability would indicate the need for a } \\
\text { full review }\end{array}$ & $\begin{array}{l}\leq 70 \% \text { discuss the generalizability of } \\
\text { findings }\end{array}$ \\
\hline
\end{tabular}

methodological review of a sample of methodological reviews published in 2017.

\section{Eligibility criteria}

We included articles that fulfilled all of the following criteria:

- Are in the domain of clinical research with human participants

- Could be classified as secondary research (i.e., investigating other studies/primary research)

- Investigate methods or reporting issues

\section{Search strategy}

We conducted a search of PubMed-a public search engine which retrieves medical literature from the MEDLINE database-from January 1, 2017 to December 31, 2017 (i.e., the most recent complete year) using terms often used to refer to MRs: "literature survey" OR "metaepidemiologic" review" OR "meta-epidemiologic" survey" OR "methodologic" review" OR "methodologic" survey" OR "systematic survey". To maintain a focused search as was intended for the scope of this pilot, phrase searching was used to restrict the volume of hits with wildcard searching.

\section{Study selection}

One reviewer (DOL) screened the titles and abstracts of retrieved articles in a reference manager program (EndNote X7.8, Philadelphia: Clarivate Analytics; 2016) for the type of study and whether any nomenclature was present in either or both the title and abstract. Studies identified as methodological reviews were screened in duplicate (DOL and AL) and verified for eligibility in the full texts using a spreadsheet (Microsoft Excel for Mac v.16.15, Washington: Microsoft Corporation; 2018).

\section{Data extraction}

Two reviewers (DOL and $\mathrm{AL}$ ) extracted data from MRs in a spreadsheet in duplicate including the first author name, country of primary affiliation (for $>1$ co-first authors, the affiliation of the first listed author was taken; for $>1$ affiliations of the first author, the senior author's affiliation was taken), nomenclature in the title, nomenclature in the abstract, nomenclature in the methods section, total number of included records (e.g., abstracts, instruments, journals, meta-analyses, reviews, and trials), if the sample size was calculated, if the authors referenced a published protocol, the databases searched for record inclusion, if there was justification of search time limits, whether the search strategy was reported (or referenced elsewhere), and if sampling of records was random.

Given our concerns with sampling issues, we sought additional information on generalizability. Generalizability was guided by the response to the following question: "Do these findings represent the total population of studies that the sample was drawn from?". We classified studies as likely generalizable if they met some or all of the following criteria:

- Used multiple databases 
- Justified their sample size (e.g., provide details for a sample size calculation)

- Selected a random sample of records (where applicable)

We classified studies as unlikely to be generalizable if they

- Used only one database

- Used selected journals (e.g., only high impact)

- Used very stringent eligibility criteria

Disagreements were resolved through discussion, and if reviewers could not come to an agreement on conflicts, a third reviewer (LM) adjudicated as necessary.

\section{Inter-rater agreement}

We assessed the level of agreement between reviewers using Cohen's kappa $(\kappa)$ for inter-rater reliability for two raters. Agreement was calculated for yes/no and numerical fields at the full-text screen and data extraction levels. The index value was interpreted as no agreement $(0-0.20)$, minimal agreement $(0.21-0.39)$, weak agreement $(0.40-0.59)$, moderate agreement $(0.60-0.79)$, strong agreement $(0.80-$ 0.90 ), and almost perfect agreement (above 0.90) [23].

\section{Data analysis}

We summarized and reported the descriptive statistics, including frequencies and percentages for count and categorical variables, and means with standard deviation (SD) for continuous variables.

In order to determine the best search strategy, we computed the sensitivity and specificity of each search term to determine which would have the best accuracy in our database of studies identified from our search. We computed the proportion of studies identified with each search term that were actually MRs (i.e., included studies and true positives) and the proportion identified that were not MRs (i.e., excluded studies and false positives). We also computed the proportion of studies not captured by the search term that were not MRs (i.e., excluded studies and true negatives) and the proportion of MRs that were not captured by the search term (i.e., included studies and false negatives). We pooled the sensitivity and specificity estimates for multiple search terms using the parallel testing approach in order to achieve an optimal combination of search terms with a good balance of sensitivity and specificity [24]. Statistical analysis was conducted in IBM SPSS Statistics (IBM SPSS Statistics v.24.0, Armonk: IBM Corporation; 2016) and inter-rater agreement ( $\kappa$ with $95 \%$ confidence interval, CI) was calculated using WinPepi [25]. We built a word cloud in WordArt to visualize common terms used for indexing MRs in PubMed [26].

\section{Ethics review}

Ethics committee approval and consent to participate was not required as this study used previously published non-human data.

\section{Results}

There were 236 articles retrieved from the PubMed search of which 31 were included in the final quantitative and qualitative analysis (see Fig. 1 for study flow diagram with reasons for exclusion) [27-57]. There was moderate inter-rater agreement between reviewers before consensus ( $\kappa=0.78$; 95\% CI 0.73-0.82).

The study characteristics of all included studies are outlined in the Appendix.

\section{Characteristics of included methodological reviews}

Many of the authors were from Canadian institutions $(n=12$, $38.7 \%)$, followed by Japan and USA ( $n=4,12.9 \%$ each). Based on the previously defined criteria, we scored ten studies $(32.3 \%)$ as generalizable [ $28,30,38,40,49,51-54,57]$. Only three studies (9.7\%, two of which we scored as generalizable) commented on generalizability and reported their own work as generalizable, either to the subject area (e.g., venous ulcer disease), to a clinical area, or in general terms $[27,30,38]$.

\section{Nomenclature}

Of the 31 included studies, $77.4 \%(n=24)$ presented the study nomenclature in their title. The terms found in the titles and abstracts of all retrieved articles that were used to describe the study are represented in Fig. 2. The most accurate search terms were "meta-epidemiological" (sensitivity [Sn] 48.39; 95\% CI 31.97-65.16; specificity [Sp] 97.56; 94.42-98.95), "systematic survey" (Sn 45.16; 95\% CI 29.1662.23; Sp 76.10; 95\% CI 69.81-81.42) "systematic review" (Sn 12.90; 95\% CI 5.13-28.85; Sp 93.17; 95\% CI 88.8695.89), and "literature survey" (Sn 6.45; 95 CI 1.79-20.72; Sp 33.66; 95\% CI 27.54-40.38), among 12 different types or combinations of nomenclature cited (Fig. 2). The combined sensitivity and specificity for the six terms attributed to MRs was $100 \%$ and $0.99 \%$, respectively.

The words "survey" and "systematic" ( $n=18$ each), "meta-epidemiologic" $(n=15)$, and "review" and "study" ( $n=7$ each) were the most frequent in the word cloud (Appendix). Five (16.1\%) of these studies used more than one name to describe their study type in the title or abstract (two names, $n=4$, and three names, $n=1$ ).

\section{Methodological features}

The mean (standard deviation [SD]) number of databases searched was 2 (1.6) with a minimum of 1 and a maximum of 8 databases. Overall, less than a quarter of studies $(n=7,22.6 \%)$ made a reference to or cited a protocol for the study. Most studies reported the search strategy $(n$ 


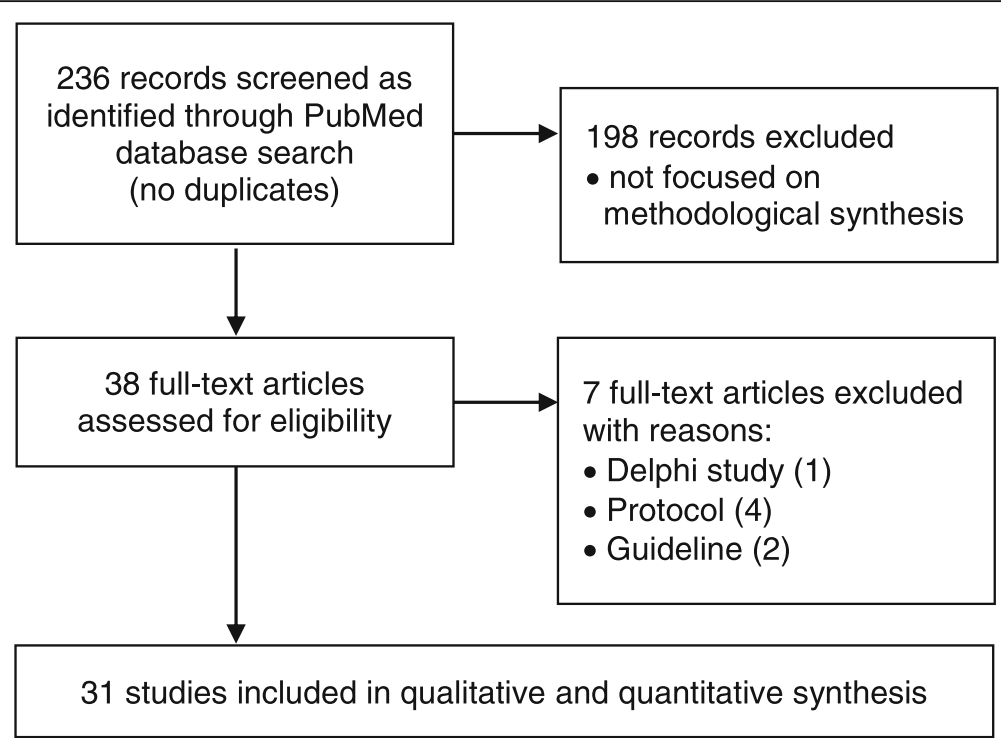

Fig. 1 Study flow diagram illustrating selection of eligible studies

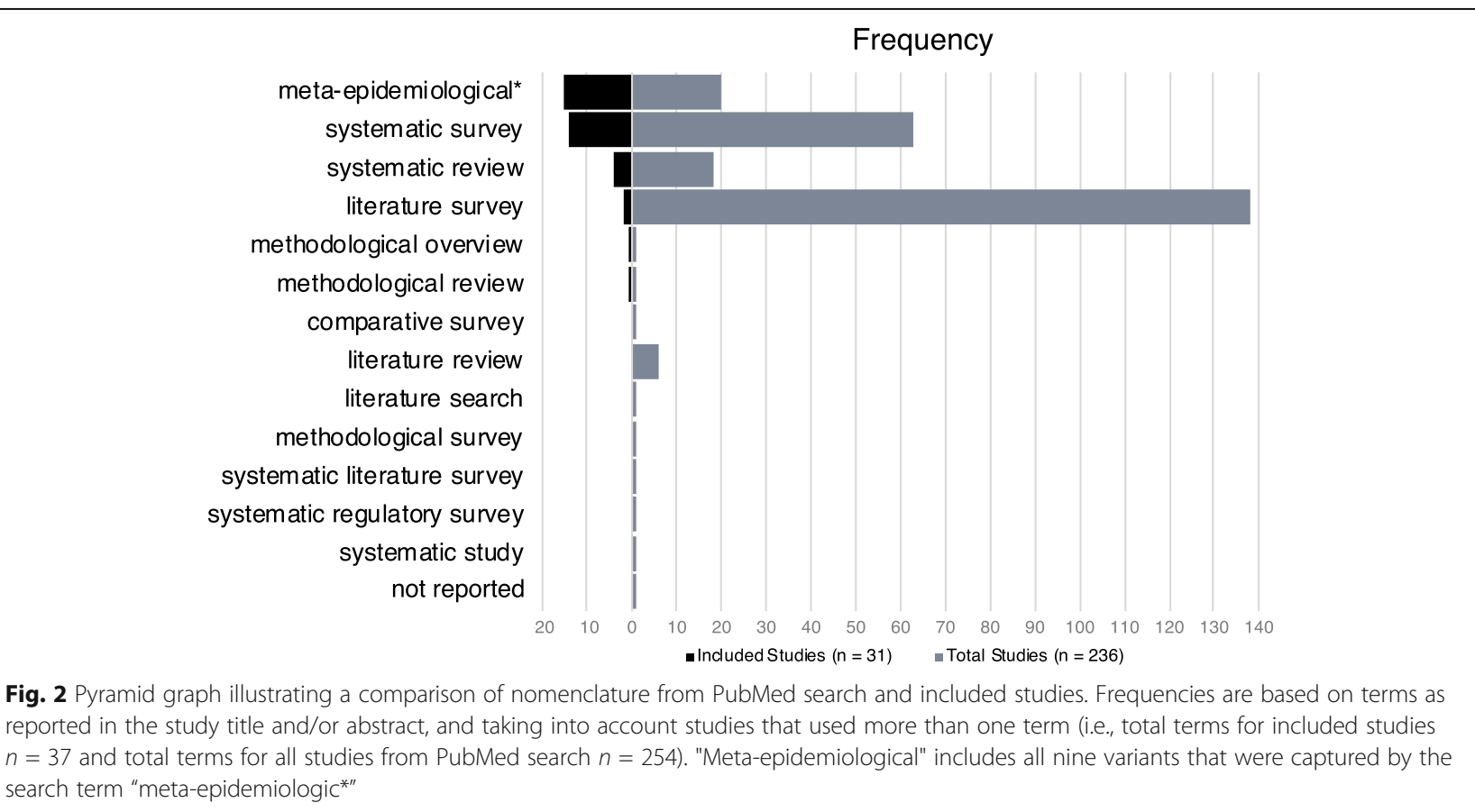


$=23,74.2 \%)$ and only eight (25.8\%) justified the time limits that were set for the search (Table 2).

Five studies (16.1\%) performed an a priori sample size calculation of records to be included, using a variety of methods. Abbade et al. used an estimate for the primary objective from a prior similar study [27], and similarly, Riado Minguez et al. used a prior similar study to determine a target sample size coupled with a power calculation [43]. El Dib et al. sampled enough studies to achieve a CI of \pm 0.10 around all proportions [33], and Zhang et al. used a precise $\mathrm{CI}$ of \pm 0.05 [57]. Kosa et al. incorporated an approach optimized for logistic regression, based on estimates for correlation between covariates [37]. Among the studies that did not aim to summarize data from all available records retrieved in their search $(n=5)$, all studies $(100 \%)$ incorporated some randomization strategy to sample the records to be included in their final synthesis [29, 33, 34, 37, 57].

\section{Discussion}

In this pilot methodological review, we have established the need for a full review and determined some of the methodological features worth investigating to facilitate the development of a reporting guideline for MRs. Unquestionably, it is highly likely that our search strategy missed MRs characterized by different nomenclature. However, as a result of this pilot we have been able to identify some of the most appropriate search terms to incorporate into a search strategy in the full review. The criteria for success of feasibility and the respective results are outlined in Table 3. The position of this pilot in the larger picture of the development of the reporting guideline is outlined in Fig. 3. Additionally, the forthcoming guideline as a result of this work, the METhodological Review reportIng Checklist (METRIC), has been registered as currently under development with EQUATOR.

The disparity in nomenclature, methods, and reporting in this assessment of 1-year worth of data suggests that a full review is required to better provide a more complete picture of the existing concerns with reporting quality. A number of key features stand out and an appraisal of these concerns would also help to inform the development of

Table 2 Methodological features of included methodological reviews $(N=31)$

\begin{tabular}{ll}
\hline Variable & $n(\%)$ \\
\hline $\begin{array}{l}\text { Reported study type (nomenclature) in the } \\
\text { "Methods" section }\end{array}$ & $12(38.7)$ \\
Number of databases searched (mean, SD) & $2(1.6)$ \\
Number of records included (median, IQR) & $77(13-1127)$ \\
Reported (or referenced) a protocol & $7(22.6)$ \\
Reported (or referenced) a search strategy & $23(74.2)$ \\
Justified search time limits & $8(25.8)$ \\
Performed a sample size calculation a priori & $5(16.1)$ \\
Randomly sampled included records (of $n=5)$ & $5(100)$ \\
\hline
\end{tabular}

$I Q R$ interquartile range, $S D$ standard deviation guidance. First, there is a growing body of literature pertaining to reviews of methods. A search of PubMed with the term "methodological review" shows that there has been a steady increase in studies indexed as MRs over the past 10 years, with ten in 2007 and 39 in 2017. The increasing number of publications addressing methodological issues in primary and secondary research would suggest that there is interest in understanding and optimizing health research methods. Therefore, the development of consensus-based guidelines in this field is warranted.

Second, there are inconsistencies in the nomenclature used to describe MRs. In the variety of names currently being attributed to MRs, nomenclature is an issue that must be addressed. This is especially true with the use of labels such as "systematic review"-which is attributed to a specific, welldefined form of evidence synthesis for healthcare studies-or in the case of some studies which have used the term "methodologic," which is not otherwise defined in English dictionaries and which could compromise their detection in searches [58].

Third, there are no methodological standards specific to MRs. Regarding selection bias, it is unclear what processes researchers employ in defining the appropriate eligibility criteria, or in the selection of databases and time periods for screening relevant literature. Readers would be interested in knowing why and how the choice of studies is ideal to answer the research question and the rationale behind such choices should be explicit. Likewise, the approaches to the sampling should be explicit, especially in methodological reviews that do not adopt systematic searches to identify and capture all of the relevant articles. For example, some methodological studies might approach a research question with a before-after, cross-sectional, or longitudinal design to name a few. As a result, it may not be appropriate or necessary to use all of the available studies in these scenarios and a sample of studies may suffice $[59,60]$.

On a conceptual level, we sought to develop a definition of study generalizability, but this was challenging to operationalize. The aim of this exercise was to help define the scope of inferences that can be made from the findings in MRs. As this area currently lacks specific guidance, we recognize that the appropriateness of the selected criteria (and its applicability to each study) will see ongoing development in subsequent investigations and may be applied differently. Generalizability is strongly tied to the target population and this was not often explicit in MRs, making any inferences challenging. Our approach to defining generalizability could be refined with insights from authors and users of MRs and will vary based on the study in question. There are several ways of addressing this outcome: do the authors identify their study as generalizable? Is the "study topic" (i.e., methodological issue) generalizable to other fields? Are the "results" generalizable to other studies in different fields investigating the same methodological issue? How are these results applicable?

Conversely, we can also consider whether the sample size and number of databases searched are surrogate indicators of 
Table 3 Feasibility results for this pilot review

\begin{tabular}{llll}
\hline Measure & Target & Observed & Description \\
\hline Sensitivity/specificity $^{\mathrm{b}}$ & $\geq 70 \%$ & $\begin{array}{l}\text { Sensitivity, 100\% } \\
\text { Specificity, 0.99\% }\end{array}$ & Six terms combined gave good sensitivity but compromised specificity \\
Published protocols $^{\mathrm{a}}$ & $\leq 70 \%$ & $22.6 \%$ & Few studies had pre-specified methods \\
Coefficient of variation $^{c}$ & $\sim 1$ & 0.8 & Fairly consistent number of databases searched \\
Justification of search limits $^{\mathrm{a}}$ & $\leq 70 \%$ & $25.8 \%$ & Few studies justified their search limits \\
Justification of sample size or perform sample size calculation $^{\mathrm{a}}$ & $\leq 70 \%$ & $16.1 \%$ & Few studies justified their sample sizes or performed calculations \\
Use a random sampling approach $^{c}$ & $\leq 70 \%$ & $100 \%$ & All studies adopted a random sampling approach \\
Discuss the generalizability of findings & & Few studies described how generalizable their findings were &
\end{tabular}

${ }^{\mathrm{a}}$ Feasibility criteria met

${ }^{b}$ Feasibility criteria partially met

'Feasibility criteria not met

generalizability. These factors could be used to extrapolate to the generalizability of an MR, as is done with clinical trials and systematic reviews, and as we have done in the present study. These questions and the importance of this variable might be answered with a deeper investigation of MRs, as well as feedback and engagement from expert users as we work to develop guidance on reporting. Through expert consensus, and recognizing typological differences, we also plan to optimize the proposed guidance for specific types of MRs (e.g., MRs assessing methods of randomized control trials or systematic reviews). We hope that these approaches will also help to tease out the appropriate definition of generalizability in each case.

\section{Conclusions}

We now have a clearer understanding of the terms used to describe methodological reviews and some of the issues that warrant a deeper investigation. In this pilot review, we have highlighted the need for a full review on this topic in order to inform future guidance for reporting methodological reviews. A full review using some of the search terms identified here is feasible. These findings will be used to develop a protocol, which will encompass more databases and years, in order to gain a clearer sense of the landscape of MRs.

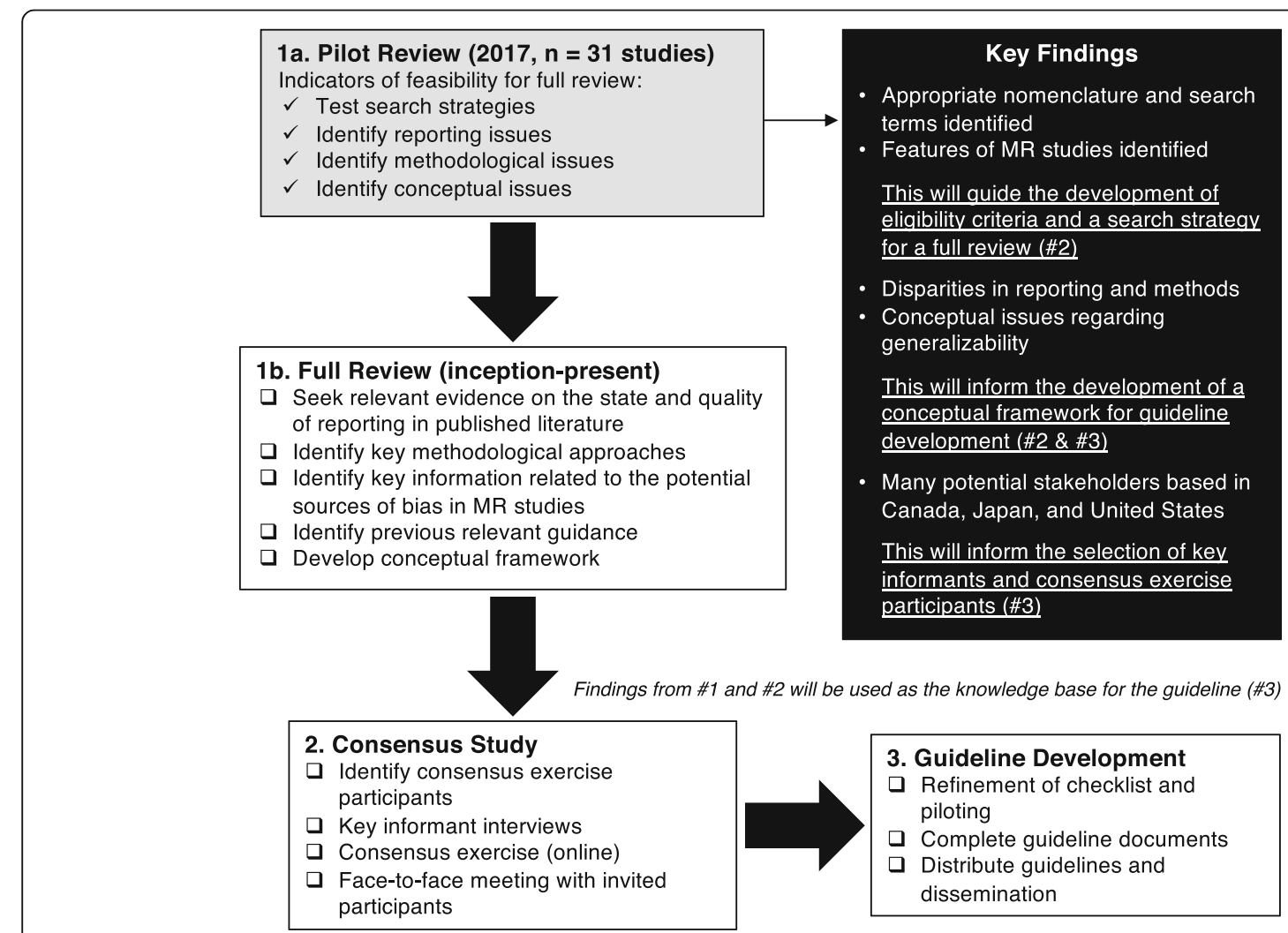

Fig. 3 Stages of development of reporting guidelines for methodological reviews 


\section{Appendix}

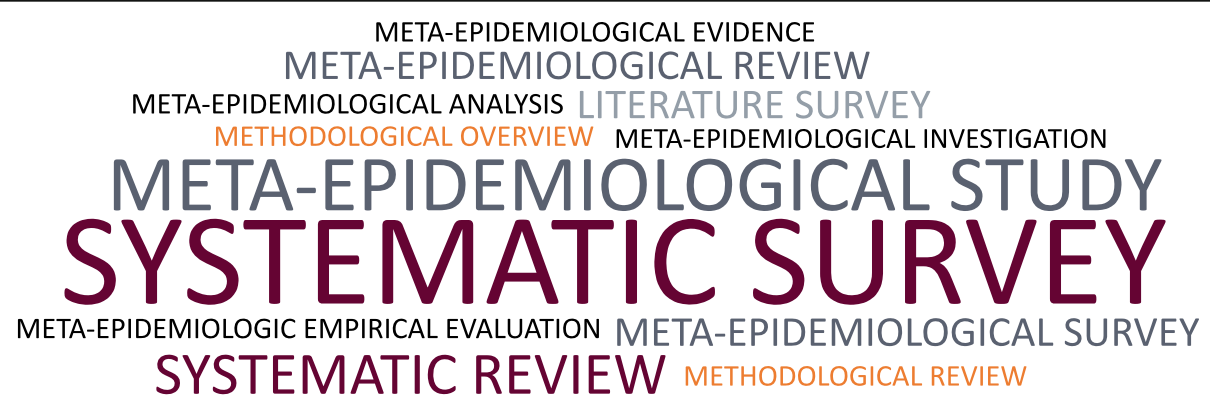

Fig. 4 Word cloud depicting frequency of PubMed MeSH terms used to describe and catalog methodological studies 
Table 4 Main characteristics of included methodological reviews $(n=31)$

\begin{tabular}{|c|c|c|c|c|c|c|c|c|c|c|}
\hline Study & Country & $\begin{array}{l}\text { Nomenclature } \\
(\mathrm{T} / \mathrm{A})\end{array}$ & $\begin{array}{l}\text { Nomenclature } \\
\text { (M) }\end{array}$ & $\begin{array}{l}\text { Databases } \\
\text { searched } \\
\text { (\#) }\end{array}$ & $\begin{array}{l}\text { Records } \\
\text { included } \\
(\#)\end{array}$ & $\begin{array}{l}\text { Reference } \\
\text { to a } \\
\text { protocol } \\
(\mathrm{Y} / \mathrm{N}) \\
\end{array}$ & $\begin{array}{l}\text { Search } \\
\text { strategy } \\
\text { reported } \\
(\mathrm{Y} / \mathrm{N}) \\
\end{array}$ & $\begin{array}{l}\text { Justification } \\
\text { of search } \\
\text { time limits } \\
(\mathrm{Y} / \mathrm{N})\end{array}$ & $\begin{array}{l}\text { Sample } \\
\text { size } \\
\text { calculation } \\
(\mathrm{Y} / \mathrm{N}) \\
\end{array}$ & $\begin{array}{l}\text { Random } \\
\text { sampling } \\
(\mathrm{Y} / \mathrm{N})\end{array}$ \\
\hline Abbade et al. [27] & Canada & SSu & SSu & 1 & 85 & Y & Y & Y & $Y(61)$ & $\mathrm{N}$ \\
\hline Abdul-Khalek et al. [28] & Lebanon & SSu & None & 2 & 57 & N & Y & N & N & N \\
\hline Armijo-Olivo et al. [29] & Canada & MES & MES & 1 & 393 & Y & Y & $\mathrm{N}$ & $\mathrm{N}$ & Y \\
\hline Bolvig et al. [30] & Denmark & MES & None & 1 & 126 & Y & Y & $\mathrm{N}$ & $\mathrm{N}$ & $\mathrm{N}$ \\
\hline $\begin{array}{l}\text { Chase Kruse and Matt } \\
\text { Vassar [31] }\end{array}$ & USA & SSu & SSu & 2 & 35 & N & $\mathrm{N}$ & $\mathrm{N}$ & $\mathrm{N}$ & $\mathrm{N}$ \\
\hline Ebrahim et al. [32] & Canada & SR/SSu & None & 3 & 28 & Y & Y & $N$ & $N$ & $\mathrm{~N}$ \\
\hline El Dib et al. [33] & Canada & SSu & None & 1 & 103 & N & Y & N & $Y(100)$ & Y \\
\hline Ge et al. [34] & China & MES & MESC & 1 & 150 & N & Y & $\mathrm{N}$ & $N$ & Y \\
\hline Gorne and Diaz [35] & Argentina & SR/SSu & LSu & 1 & 128 & N & Y & N & $N$ & $N$ \\
\hline Khan et al. [36] & Canada & SSu & SSu & 3 & 48 & N & Y & N & $N$ & N \\
\hline Kosa et al. [37] & Canada & MR, SSu & $M R$ & 1 & 200 & N & Y & Y & Y (152) & Y \\
\hline Kovic et al. [38] & Canada & MESU & MES, SR & 2 & 77 & N & Y & Y & N & $N$ \\
\hline Kuriyama et al. [39] & Japan & LSu & None & 1 & 353 & N & N & Y & $N$ & $\mathrm{~N}$ \\
\hline Manja et al. [40] & Canada & SSu & None & 5 & 43 & N & Y & N & N & N \\
\hline $\begin{array}{l}\text { Papageorgiou et al. } \\
\text { [41] }\end{array}$ & Switzerland & MEE, MO, SR & $S R$ & 8 & 34 & Y & Y & N & $N$ & $\mathrm{~N}$ \\
\hline Ratib et al. [42] & UK & MER & None & 1 & 1127 & N & N & N & N & $\mathrm{N}$ \\
\hline $\begin{array}{l}\text { Riado Minguez et al. } \\
\text { [43] }\end{array}$ & Spain & MES & None & 1 & 446 & N & Y & Y & $Y(150)$ & $\mathrm{N}$ \\
\hline Sekercioglu et al. [44] & Canada & SSu & None & 5 & 16 & N & Y & $\mathrm{N}$ & $\mathrm{N}$ & $\mathrm{N}$ \\
\hline Shinohara et al. [45] & Japan & $\mathrm{MEl}, \mathrm{SR}$ & None & 1 & 60 & Y & Y & $\mathrm{N}$ & $\mathrm{N}$ & $\mathrm{N}$ \\
\hline Sims et al. [46] & USA & MES & MES, Su & 1 & 37 & Y & N & $\mathrm{N}$ & $N$ & $\mathrm{~N}$ \\
\hline Storz-Pfennig [47] & Germany & MEA & None & 1 & 13 & N & N & $\mathrm{N}$ & $\mathrm{N}$ & $N$ \\
\hline Tedesco et al. [48] & Italy & MEEE & None & 1 & 244 & N & $\mathrm{N}$ & $\mathrm{N}$ & $\mathrm{N}$ & $\mathrm{N}$ \\
\hline Tsujimoto et al. [49] & Japan & MES & None & 3 & 326 & N & N & Y & $N$ & $N$ \\
\hline Tsujimoto et al. [50] & Japan & MES & None & 1 & 284 & N & Y & Y & $N$ & $N$ \\
\hline Umberham et al. [51] & USA & MER & None & 2 & 265 & N & Y & $\mathrm{N}$ & $N$ & $N$ \\
\hline $\begin{array}{l}\text { von Niederhausern } \\
\text { et al. [52] }\end{array}$ & Switzerland & SSu & None & 2 & 47 & N & Y & $\mathrm{N}$ & $\mathrm{N}$ & $\mathrm{N}$ \\
\hline Wallach et al. [53] & USA & MESU & MESU & 3 & 64 & N & N & $\mathrm{N}$ & $\mathrm{N}$ & $\mathrm{N}$ \\
\hline Yepes-Nunez et al. [54] & Canada & SSu & None & 3 & 42 & N & Y & $\mathrm{N}$ & $\mathrm{N}$ & $\mathrm{N}$ \\
\hline Yu et al. [55] & Taiwan & LSu & LSu of SRs & 1 & 29 & N & Y & $\mathrm{N}$ & $\mathrm{N}$ & $\mathrm{N}$ \\
\hline Zhang et al. [56] & Canada & SSu & None & 4 & 60 & N & Y & $\mathrm{N}$ & N & $\mathrm{N}$ \\
\hline Zhang et al. [57] & Canada & SSu & None & 1 & 200 & N & Y & Y & Y (200) & Y \\
\hline
\end{tabular}

LSU literature survey, $M$ methods section, MEA meta-epidemiological analysis, MEE meta-epidemiological evidence, $M E E E$ meta-epidemiologic empirical evaluation, MEI meta-epidemiological investigation, MER meta-epidemiological review, MES meta-epidemiological study, MESc comparative meta-epidemiological study, MESu meta-epidemiological survey, MO methodological overview, MR methodological review, $N$ no, SR systematic review, SSu systematic survey, Su survey, T/A title or abstract section, UK United Kingdom, USA United States of America, $Y$ yes 


\section{Abbreviations}

Cl: Confidence interval; CONSORT: Consolidated Standards of Reporting Trials; EQUATOR: Enhancing the QUAlity and Transparency Of health Research; IQR: Interquartile range; MeSH: Medical Subject Headings; MR: Methodological review or review of methods; N/n: Sample size; PRISMA: Preferred Reporting Items for Systematic Reviews and MetaAnalyses; SD: Standard deviation

\section{Acknowledgements}

We thank Professor Lehana Thabane for his editorial support and helpful feedback on drafts of this paper.

\section{Authors' contributions}

LM and DOL conceptualized and designed the study. DOL, AL, and LM collected, analyzed, and interpreted the data. DOL wrote the first draft of the manuscript. DOL, LM, and AL contributed to the critical revision of the manuscript, and all authors read and approved the final version.

\section{Funding}

No funding sources supported the conduct of this research.

\section{Availability of data and materials}

In addition to the supplementary information files which are included in this published article, the dataset generated and analyzed during the current study is available from the corresponding author on reasonable request.

\section{Ethics approval and consent to participate}

Not applicable-ethics committee approval and consent to participate was not required as this study used previously published data.

\section{Consent for publication}

Not applicable

\section{Competing interests}

The authors declare that they have no competing interests.

\section{Author details}

'Department of Health Research Methods, Evidence, and Impact, McMaster University, 1280 Main Street West, Hamilton, ON L8S 4K1, Canada. ${ }^{2}$ Faculty of Health Sciences, McMaster University, 1280 Main Street West, Hamilton, ON L8S 4K1, Canada. 'Biostatistics Unit, Father Sean O'Sullivan Research Centre, St. Joseph's Healthcare Hamilton, Hamilton, ON L8N 4A6, Canada.

Received: 24 February 2019 Accepted: 20 December 2019 Published online: 30 January 2020

\section{References}

1. Guyatt GH, Rennie D, Meade MO, Cook DJ, American Medical Association JAMA Network. Users' guides to the medical literature: a manual for evidence-based clinical practice. In: Third edition edn, vol. xxv; 2015. p. 697.

2. Hulley SB. Designing clinical research. 4th ed. Philadelphia: Wolters Kluwer/ Lippincott Williams \& Wilkins; 2013.

3. Dwan K, Altman DG, Cresswell L, Blundell M, Gamble CL, Williamson PR Comparison of protocols and registry entries to published reports for randomised controlled trials. Cochrane Database Syst Rev. 2011;1:MR000031.

4. Ghimire S, Kyung E, Kang W, Kim E. Assessment of adherence to the CONSORT statement for quality of reports on randomized controlled trial abstracts from four high-impact general medical journals. Trials. 2012;13:77.

5. Ma J, Akhtar-Danesh N, Dolovich L, Thabane L, investigators C. Imputation strategies for missing binary outcomes in cluster randomized trials. BMC Med Res Methodol. 2011:11:18.

6. Mbuagbaw L, Rochwerg B, Jaeschke R, Heels-Andsell D, Alhazzani W, Thabane L, Guyatt GH. Approaches to interpreting and choosing the best treatments in network meta-analyses. Syst Rev. 2017;6(1):79.

7. Mbuagbaw L, Thabane M, Vanniyasingam T, Borg Debono V, Kosa S, Zhang S, Ye C, Parpia S, Dennis BB, Thabane L. Improvement in the quality of abstracts in major clinical journals since CONSORT extension for abstracts: a systematic review. Contemp Clin Trials. 2014;38(2):245-50.

8. Schulz KF, Altman DG, Moher D, Group C. CONSORT 2010 statement: updated guidelines for reporting parallel group randomised trials. BMJ. 2010;340:c332
9. The EQUATOR Network: Enhancing the QUAlity and Transparency Of health Research [http://www.equator-network.org]

10. Moher D, Schulz KF, Simera I, Altman DG. Guidance for developers of health research reporting guidelines. PLoS Med. 2010;7(2):e1000217.

11. Jin Y, Sanger N, Shams I, Luo C, Shahid H, Li G, Bhatt M, Zielinski L, Bantoto $B$, Wang $M$, et al. Does the medical literature remain inadequately described despite having reporting guidelines for 21 years? - A systematic review of reviews: an update. J Multidiscip Healthc. 2018;11:495-510.

12. Moher D, Jones A, Lepage L, Group C. Use of the CONSORT statement and quality of reports of randomized trials: a comparative before-and-after evaluation. JAMA. 2001;285(15):1992-5.

13. Umbrella reviews: evidence synthesis with overviews of reviews and metaepidemiologic studies. New York: Springer Berlin Heidelberg; 2016.

14. Murad MH, Wang Z. Guidelines for reporting meta-epidemiological methodology research. Evidence-based medicine. 2017;22(4):139-42.

15. Moher D, Liberati A, Tetzlaff J, Altman DG, Group P. Preferred reporting items for systematic reviews and meta-analyses: the PRISMA statement. PLoS Med. 2009;6(7):e1000097.

16. Ivers NM, Taljaard M, Dixon S, Bennett C, McRae A, Taleban J, Skea Z, Brehaut JC, Boruch RF, Eccles MP, et al. Impact of CONSORT extension for cluster randomised trials on quality of reporting and study methodology: review of random sample of 300 trials, 2000-8. BMJ. 2011;343:d5886.

17. Hopewell S, Ravaud P, Baron G, Boutron I. Effect of editors' implementation of CONSORT guidelines on the reporting of abstracts in high impact medical journals: interrupted time series analysis. BMJ. 2012;344:e4178.

18. Shanthanna H, Kaushal A, Mbuagbaw L, Couban R, Busse J, Thabane L. A cross-sectional study of the reporting quality of pilot or feasibility trials in high-impact anesthesia journals. Can J Anaesth. 2018:65(11):1180-95.

19. Tanniou J, van der Tweel I, Teerenstra S, Roes KC. Subgroup analyses in confirmatory clinical trials: time to be specific about their purposes. BMC Med Res Methodol. 2016;16:20

20. Puljak L. Reporting checklist for methodological, that is, research on research studies is urgently needed. J Clin Epidemiol. 2019;112:93.

21. Long L. Routine piloting in systematic reviews--a modified approach? Syst Rev. 2014;3:77.

22. Newman M, Van den Bossche P, Gijbels D, McKendree J, Roberts T, Rolfe I, Smucny J, De Virgilio G. Campbell Collaboration Review Group on the effectiveness of problem-based $L$ : responses to the pilot systematic review of problem-based learning. Med Educ. 2004;38(9):921-3.

23. McHugh ML. Interrater reliability: the kappa statistic. Biochem Med (Zagreb). 2012;22(3):276-82

24. Cebul RD, Hershey JC, Williams SV. Using multiple tests: series and parallel approaches. Clin Lab Med. 1982;2(4):871-90.

25. Abramson JH. WINPEPI updated: computer programs for epidemiologists, and their teaching potential. Epidemiol Perspect Innov. 2011;8(1):1.

26. WordArt.com: Word cloud art creator [https://www.wordart.com]

27. Abbade LPF, Wang M, Sriganesh $K$, Jin $Y$, Mbuagbaw L, Thabane L. The framing of research questions using the PICOT format in randomized controlled trials of venous ulcer disease is suboptimal: a systematic survey. Wound Repair Regen. 2017;25(5):892-900

28. Abdul-Khalek RA, Darzi AJ, Godah MW, Kilzar L, Lakis C, Agarwal A, Abou-Jaoude E, Meerpohl J, Wiercioch W, Santesso N, et al. Methods used in adaptation of health-related quidelines: A systematic survey. J Glob Health. 2017:7(2):020412.

29. Armijo-Olivo S, Fuentes J, da Costa BR, Saltaji H, Ha C, Cummings GG. Blinding in physical therapy trials and its association with treatment effects: a meta-epidemiological study. Am J Phys Med Rehabil. 2017;96(1):34-44.

30. Bolvig J, Juhl CB, Boutron I, Tugwell P, Ghogomu EAT, Pardo JP, Rader T, Wells GA, Mayhew A, Maxwell L, et al. Some Cochrane risk of bias items are not important in osteoarthritis trials: a meta-epidemiological study based on Cochrane reviews. J Clin Epidemiol. 2017.

31. Chase Kruse B, Matt Vassar B. Unbreakable? An analysis of the fragility of randomized trials that support diabetes treatment guidelines. Diabetes Res Clin Pract. 2017;134:91-105

32. Ebrahim S, Vercammen $\mathrm{K}$, Sivanand A, Guyatt GH, Carrasco-Labra A, Fernandes RM, Crawford MW, Nesrallah G, Johnston BC. Minimally important differences in patient or proxy-reported outcome studies relevant to children: a systematic review. Pediatrics. 2017;139:3.

33. El Dib R, Tikkinen KAO, Akl EA, Gomaa HA, Mustafa RA, Agarwal A, Carpenter $C R$, Zhang $Y$, Jorge EC, Almeida $R$, et al. Systematic survey of randomized trials evaluating the impact of alternative diagnostic strategies on patientimportant outcomes. J Clin Epidemiol. 2017;84:61-9. 
34. Ge L, Tian JH, Li YN, Pan JX, Li G, Wei D, Xing X, Pan B, Chen YL, Song FJ, et al. Association between prospective registration and overall reporting and methodological quality of systematic reviews: a meta-epidemiological study. J Clin Epidemiol. 2018;93:45-55.

35. Gorne LD, Diaz S. A novel meta-analytical approach to improve systematic review of rates and patterns of microevolution. Ecol Evol. 2017;7(15):5821-32.

36. Khan M, Evaniew N, Gichuru M, Habib A, Ayeni OR, Bedi A, Walsh M, Devereaux PJ, Bhandari M. The fragility of statistically significant findings from randomized trials in sports surgery: a systematic survey. Am J Sports Med. 2017:45(9):2164-70.

37. Kosa SD, Mbuagbaw L, Debono VB, Bhandari M, Dennis BB, Ene G, Leenus A, Shi D, Thabane M, Valvasori S, et al. Agreement in reporting between trial publications and current clinical trial registry in high impact journals: a methodological review. Contemp Clin Trials. 2017;65:144-50.

38. Kovic B, Zoratti MJ, Michalopoulos S, Silvestre C, Thorlund K, Thabane L. Deficiencies in addressing effect modification in network meta-analyses: a meta-epidemiological survey. J Clin Epidemiol. 2017;88:47-56.

39. Kuriyama A, Takahashi N, Nakayama T. Reporting of critical care trial abstracts: a comparison before and after the announcement of CONSORT guideline for abstracts. Trials. 2017;18(1):32.

40. Manja V, AlBashir S, Guyatt G. Criteria for use of composite end points for competing risks-a systematic survey of the literature with recommendations. J Clin Epidemiol. 2017;82:4-11.

41. Papageorgiou SN, Hochli D, Eliades T. Outcomes of comprehensive fixed appliance orthodontic treatment: A systematic review with meta-analysis and methodological overview. Korean J Orthod. 2017;47(6):401-13.

42. Ratib S, Wilkes SR, Grainge MJ, Thomas KS, Tobinska C, Williams HC. Is there an association between study size and reporting of study quality in dermatological clinical trials? A meta-epidemiological review. Br J Dermatol. 2017;176(6):1657-8.

43. Riado Minguez D, Kowalski M, Vallve Odena M, Longin Pontzen D, Jelicic Kadic A, Jeric M, Dosenovic S, Jakus D, Vrdoljak M, Poklepovic Pericic T, et al. Methodological and reporting quality of systematic reviews published in the highest ranking journals in the field of pain. Anesth Analg. 2017;125(4):1348-54.

44. Sekercioglu N, Al-Khalifah R, Ewusie JE, Elias RM, Thabane L, Busse JW, AkhtarDanesh N, lorio A, Isayama T, Martinez JP, et al. A critical appraisal of chronic kidney disease mineral and bone disorders clinical practice guidelines using the AGREE II instrument. Int Urol Nephrol. 2017;49(2):273-84.

45. Shinohara K, Suganuma AM, Imai H, Takeshima N, Hayasaka Y, Furukawa TA. Overstatements in abstract conclusions claiming effectiveness of interventions in psychiatry: a meta-epidemiological investigation. PLoS One. 2017;12(9):e0184786

46. Sims MT, Checketts JX, Wayant C, Vassar M. Requirements for trial registration and adherence to reporting guidelines in critical care journals: a meta-epidemiological study of journals' instructions for authors. Int J Evid Based Healthc. 2017

47. Storz-Pfennig P. Potentially unnecessary and wasteful clinical trial research detected in cumulative meta-epidemiological and trial sequential analysis. J Clin Epidemiol. 2017:82:61-70.

48. Tedesco D, Farid-Kapadia M, Offringa M, Bhutta ZA, Maldonado Y, loannidis JPA, Contopoulos-loannidis DG. Comparative evidence on harms in pediatric RCTs from less developed versus more developed countries is limited. J Clin Epidemiol. 2017.

49. Tsujimoto H, Tsujimoto Y, Kataoka Y. Unpublished systematic reviews and financial support: a meta-epidemiological study. BMC Res Notes. 2017;10(1):703.

50. Tsujimoto Y, Tsujimoto H, Kataoka Y, Kimachi M, Shimizu S, Ikenoue T, Fukuma S, Yamamoto $Y$, Fukuhara S. Majority of systematic reviews published in high-impact journals neglected to register the protocols: a meta-epidemiological study. J Clin Epidemiol. 2017;84:54-60.

51. Umberham B, Hedin R, Detweiler B, Kollmorgen L, Hicks C, Vassar M. Heterogeneity of studies in anesthesiology systematic reviews: a metaepidemiological review and proposal for evidence mapping. Br J Anaesth. 2017;119(5):874-84.

52. von Niederhausern B, Schandelmaier S, Mi Bonde M, Brunner N, Hemkens LG, Rutquist M, Bhatnagar N, Guyatt GH, Pauli-Magnus C, Briel M. Towards the development of a comprehensive framework: qualitative systematic survey of definitions of clinical research quality. PLoS One. 2017;12(7): e0180635.

53. Wallach JD, Sullivan PG, Trepanowski JF, Sainani KL, Steyerberg EW, loannidis JP. Evaluation of evidence of statistical support and corroboration of subgroup claims in randomized clinical trials. JAMA Int Med. 2017;177(4):554-60.
54. Yepes-Nunez JJ, Zhang Y, Xie F, Alonso-Coello P, Selva A, Schunemann H, Guyatt G. Forty-two systematic reviews generated 23 items for assessing the risk of bias in values and preferences' studies. J Clin Epidemiol. 2017:85:21-31.

55. Yu T, Enkh-Amgalan N, Zorigt G. Methods to perform systematic reviews of patient preferences: a literature survey. BMC Med Res Methodol. 2017;17(1):166.

56. Zhang Y, Alyass A, Vanniyasingam T, Sadeghirad B, Florez ID, Pichika SC, Kennedy SA, Abdulkarimova U, Zhang Y, Iljon T, et al. A systematic survey of the methods literature on the reporting quality and optimal methods of handling participants with missing outcome data for continuous outcomes in randomized controlled trials. J Clin Epidemiol. 2017;88:67-80.

57. Zhang Y, Florez ID, Colunga Lozano LE, Aloweni FAB, Kennedy SA, Li A, Craigie S, Zhang S, Agarwal A, Lopes LC, et al. A systematic survey on reporting and methods for handling missing participant data for continuous outcomes in randomized controlled trials. J Clin Epidemiol. 2017;88:57-66.

58. In: Oxford Dictionaries: English (online). Oxford: Oxford University Press; 2018.

59. Page MJ, Altman DG, McKenzie JE, Shamseer L, Ahmadzai N, Wolfe D, Yazdi F, Catala-Lopez F, Tricco AC, Moher D. Flaws in the application and interpretation of statistical analyses in systematic reviews of therapeutic interventions were common: a cross-sectional analysis. J Clin Epidemiol. 2018;95:7-18

60. Page MJ, Shamseer $L$, Tricco AC. Registration of systematic reviews in PROSPERO: 30,000 records and counting. Syst Rev. 2018;7(1):32.

\section{Publisher's Note}

Springer Nature remains neutral with regard to jurisdictional claims in published maps and institutional affiliations.
Ready to submit your research? Choose BMC and benefit from:

- fast, convenient online submission

- thorough peer review by experienced researchers in your field

- rapid publication on acceptance

- support for research data, including large and complex data types

- gold Open Access which fosters wider collaboration and increased citations

- maximum visibility for your research: over $100 \mathrm{M}$ website views per year

At BMC, research is always in progress.

Learn more biomedcentral.com/submissions 